\title{
EXTENDED SURVEYS OF MC STAR CLUSTERS
}

\author{
M. KONTIZAS ${ }^{1}$ and E. KONTIZAS ${ }^{2}$ \\ ${ }^{1}$ Section of Astrophysics, Astronomy and Mechanics, Department of Physics, \\ University of Athens, Greece \\ ${ }^{2}$ Astronomical Institute, National Observatory of Athens, P.O. Box 20 048, Thission, \\ Athens, Gr-118 10, Greece
}

\begin{abstract}
The location of the LMC clusters, their age distribution and dynamical parameters have shown that two star cluster systems exist in this galaxy: 1) one extended system, elliptical in shape, which contains all intermediate and old star clusters and 2) another system, again elliptical, but smaller in diameter, superimposed on the other and inclined by about 50 degrees from the latter. It contains the most massive, very young globular clusters which are still at their early phases of dynamical evolution. The existence of very rich stellar associations, binary star clusters and very elliptical ones located in this system, indicates that massive star clusters have formed very recently and are still under formation.
\end{abstract}

\section{Introduction}

The star cluster system or systems provide a powerful tool for studying the stellar evolution, dynamics and the formation history of a galaxy. In our galaxy there are about 157 globular clusters which form two separate subsystems, a slowly rotating halo and a rapidly rotating disc. The stellar content of both systems is indistinguishable from that of their adjoining fields. The metallicity cut-off accepted is at $\approx-0.8$ for the metal rich disk ones whereas the metal poorer stars belong to the halo system (Zinn 1990). The open clusters of our galaxy are found in the galactic disk and do not seem to form in a different way from the globulars.

In the LMC, the expected total number of star clusters of all ages is about 4319 (Kontizas et al. 1988, 1990; Hodge 1988). Freeman et al. (1993) and later Schommer (1991) have found that the young and intermediate clusters follow the general rotation of the LMC while the oldest ones are located in another flattened system inclined by $\approx 50^{\circ}$. The distribution of the clusters (Kontizas et al. 1990) appears to show two subsystems: an outer ellipse of low surface density (the outer system) and an inner ellipse of high density (the inner system). The outer system extends over $13.2^{\circ}$ along its major axis, whereas the inner one extends over $\approx 8^{\circ}$. These two subsystems are asymmetrically superimposed.

The aim of this work is to discuss the existence of these two systems and give the properties of their cluster members. 


\section{Observational Material and Reductions}

The investigations for a large number of star clusters are based on several spectroscopic and direct photographic plates taken with the $1.2 \mathrm{~m}$ U.K. Schmidt Telescope. Objective prism plates of medium ( $840 \AA / \mathrm{mm}$ at $\mathrm{H} \gamma)$ and low $(2400 \AA / \mathrm{mm}$ at $\mathrm{H} \gamma)$ dispersion spectra were used to examine the spectral types of stars in the most populous star clusters all over the LMC area. The distribution of spectral types corrected for the field stars were used to study the stellar content of the clusters down to $B=18.5$ mag (Kontizas et al. 1987). Direct plates in various colours and exposure times reaching up to $\mathrm{V} \approx 21.5 \mathrm{mag}$ were used for star counts in order to study the dynamical parameters (Chrysovergis et al. 1989) and morphology of star clusters and associations (Kontizas et al. 1989; Argyropoulos et al. 1993).

\section{Discussion}

The stellar systems of the LMC present a very wide range of ages, morphological structure, chemical abundances, central densities, being not only single but very much in binary systems.

\subsection{STELLAR CONTENT AND DYNAMICAL PARAMETERS}

From our study of spectral types of stars for more than 75 clusters and HR diagrams from previous investigators it is found that there are globular clusters of all ages in the LMC and maybe some are still forming (Kontizas 1991).

Over 200 clusters have been studied by means of star counts, and conventional King models were used to derive their dynamical parameters from their radial density profiles. In most cases, the isotropic truncated model describes well the observed profiles. The clusters were divided into two categories according to the total number of stars:

a) Populous globulars ( $>10^{4}$ stars). These clusters belong to all age groups from $10^{7}$ yr to very old. Their dynamical behaviour indicates a very wide range of dynamical evolutionary stages. (i) The youngest clusters are the most massive and extended (tidal radii 60-80 pc) ones and are expected to relax in about $10^{\circ}$ yr if we assume a two-body encounter mechanism. However their stellar population is much younger so their dynamical stage must be at a very early phase when only the violent relaxation had time to affect the clusters' profiles (Chrysovergis 1991). These clusters are located in the inner system. (ii) The very populous clusters with intermediate and old stellar content (tidal radii $<60 \mathrm{pc}$ and age $>5 \times 10^{8} \mathrm{yr}$ ) are old enough to have relaxed profiles through the two body relaxation process. These clusters are found all over the LMC outer system.

b) Small clusters with $\mathbf{N}<10^{4}$ stars (open). The tidal radii of 70 small clusters were found to be 14 to $37 \mathrm{pc}$. Their total dynamical masses are found to be within $10^{3}$ to $10^{4} \mathrm{M}_{0}$ and give a very good extrapolation to the LMC rotation curve (Freeman 1970). Considering the latter and their spatial distribution, we conclude that the small clusters of the LMC are part of the outer disk which seems to fit the exponential disk and extends to much larger distances than calculated (Freeman 1970). From the open clusters, 80\% seem to fit the King model and have relaxed in the conventional two body relaxation mechanism. The remaining $20 \%$ of them either do not reach a flat central density, or they show deviations from the King profile 
at large distances from the centre. A small group of clusters (8) exhibit a 'bump' in their density profile which is explained either due to the weak gravitational attraction of the parent galaxy, or that these clusters are in the stage of core oscillation (Metaxa 1992).

It is found that the frequency distribution of the tidal radii of the very young populous clusters is statistically different from that of the open and old relaxed clusters with a probability of $99 \%$. This is evidence for the existence of a separate globular cluster system in the LMC central region. From the derived core and tidal radii we calculated the half mass radii and their total masses which can give us their central densities. The clusters form two groups according to their densities. About 13 associations have also been measured by means of star counts and their median radii were calculated. HR diagrams and spectral classification (Lucke 1972; Argyropoulos 1993) were used to derive their total masses and their central densities. Figure 1 shows the frequency distributions of the central densities for the three groups. It is obvious that there is a strong difference in the observed histograms and the three categories are completely discrete. The studied associations are stellar groups which will soon die, whereas the small and populous clusters are bound systems.

The same result is illustrated if we adopt the relation introduced by Geyer \& Hopp (1980) between the number of stars $\mathbf{N}$ included in the cluster's radius $\mathbf{R}$ (Fig. 2). Our values for the associations are also added in Fig 2. There is a definite split between the open (squares), populous (stars) clusters and the associations (crosses) but the linear relation of total mass vs. radius has the same slope.

\subsection{MORPHOLOGY}

The frequency distribution of ellipticities for 84 stars clusters has shown (Kontizas et al. 1989) that the youngest and more massive clusters tend to be flatter than the older ones. Therefore the

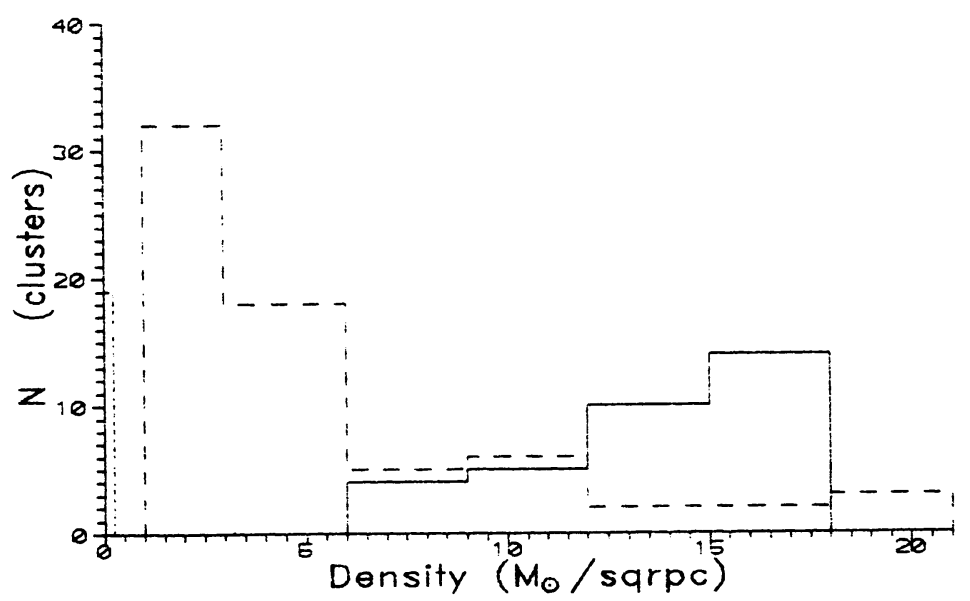

Figure 1. Frequency distribution of the central densities of the LMC stellar systems (a) for the associations (dotted line) (b) for the small clusters (dashed line) and (c) for the rich globulars (solid line). 


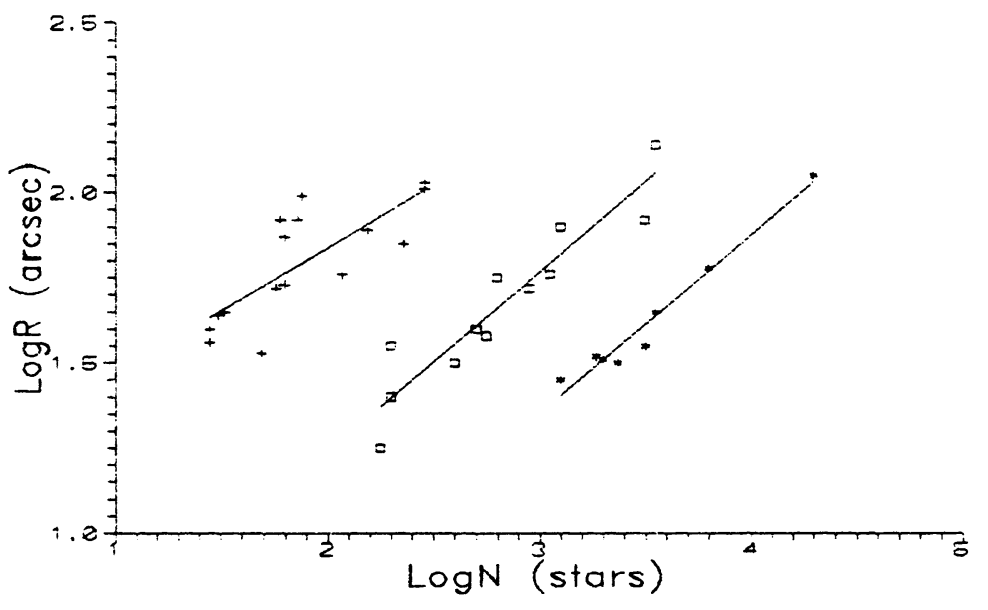

Figure 2. The total number of stars vs. radius for (a) the stellar associations (crosses) (b) the small (open) clusters (squares) and (c) the rich globulars (stars) of the LMC.

most elliptical clusters are located in the inner system.

\subsection{METALLICITY}

Assuming that two cluster systems exist, a systematic radial trend of metallicity is found for the outer system, whereas no such trend exists for the inner system where all clusters are systematically more metal rich than -1.2 dex (Kontizas et al. 1992).

Summarizing, the youngest metal rich stellar systems of this galaxy are GLOBULAR clusters, which with the associations are located in the inner system (disk?), whereas all other clusters (open and globulars) seem well distributed in the large outer system (exponential disk).

\section{Acknowledgments}

The authors are very much obliged to the $1.2 \mathrm{~m}$ UK Schmidt Telescope Unit for kindly providing the observation material all these years.

\section{References}

Argyropoulos, S., Kontizas, E., Kontizas, M. and Dapergolas, A., 1993. In preparation.

Chrysovergis, M., Kontizas, M. and Kontizas E., 1989. Astron. \& Astrophys. Suppl., 77, 357.

Chrysovergis M., 1991. PhD Thesis, Athens University.

Freeman, K.C., 1970. Astrophys. J., 160, 811.

Freeman, K.C., Illingworth, G. and Oemler, A., 1983. Astron. J., 272, 488.

Geyer, E.H. and Hopp, U., 1980. Astroph. \& Sp. Sci. 69, 495. 
Hodge, P., 1988. Publ. Astron. Soc. Pacific, 100, 1051.

Kontizas, E., Kontizas, M. and Xiradaki, E., 1987a. Astron. \& Astrophys. Suppl., 71, 575.

Kontizas, E., Metaxa, M. and Kontizas, M., 1988. Astron. J., 96, 1625.

Kontizas, E., Kontizas, M., Sedmak, G. and Smareglia, R., 1989. Astron. J., 98, 590.

Kontizas, M., Morgan, D.H., Hadzidimitriou D. and Kontizas, E., 1990. Astron. \& Astrophys. Suppl., 84, 257.

Kontizas, M. and Kontizas, E., 1991. In 'Formation and Evolution of Star Clusters', Astronomical Society of the Pacific Conference Series, ed. K. A. Janes, p. 407.

Schommer, R.A., 1991. In: 'The Magellanic Clouds', Proc. IAU Symp. 148, eds. R. Haynes, D. Milne, p. 132.

Zinn, R., 1990. Roy Astr. Soc. Con., Vol. 84, 89. 